\title{
Goal-Striving Stress, Social Economic Status, and the Mental Health of Black Americans
}

\author{
SHERRILL L. SELLERS ${ }^{a}$ AND HAROLD W. NEIGHBORS ${ }^{b}$ \\ ${ }^{a}$ School of Social Work, Florida State University, Tallahassee, Florida 32306, USA \\ ${ }^{b}$ School of Public Health and Institute for Social Research, \\ University of Michigan, Ann Arbor, Michigan 48106, USA
}

\section{INTRODUCTION}

Goal-striving stress refers to the discrepancy between aspirations and achievements, hard work and accomplishment, options and opportunities, and perhaps provides a pathway linking social structure and mental health. Although a number of scholars have speculated about the relationship between blocked opportunities and psychopathology, few studies have empirically examined associations between striving efforts and mental health among black Americans. ${ }^{1-5}$ In addition, existing studies have often used community samples, considered a single aspect of mental health, or were unable to operationalize goal-striving stress in a more nuanced fashion. ${ }^{2,4}$ This study examines the influence of goal-striving stress on the mental health of a national sample of black Americans.

\section{METHODS}

Data

Data were drawn from the second wave of data collection of the National Survey of Black Americans (NSBA). ${ }^{3}$ Of the original 2,107 respondents, 951 were reinterviewed in 1987 (approximately 82\% of those who were located).

\section{Dependent variables}

Three dimensions of mental health were considered: well-being, self-esteem, and psychological distress. Well-being was measured by happiness, a single item that ranged from "very happy" to "not happy at all" on a three-point scale, and life satisfaction, a single item that ranged from high satisfaction to low satisfaction on a fourpoint scale. A self-esteem index was constructed from six items that assessed respondents' sense of self-worth, with high scores indicating high self-esteem. A 10-item scale of psychological distress was used, with high scores indicating higher levels of distress.

\section{Independent variables}

Poverty status was measured by dichotomizing an income-to-needs score. Goalstriving stress consisted of three items measuring the discrepancy between aspirations and achievement weighted by the level of disappointment associated with failing to achieve one's goals. Respondents were asked to imagine a ladder with 10 steps 


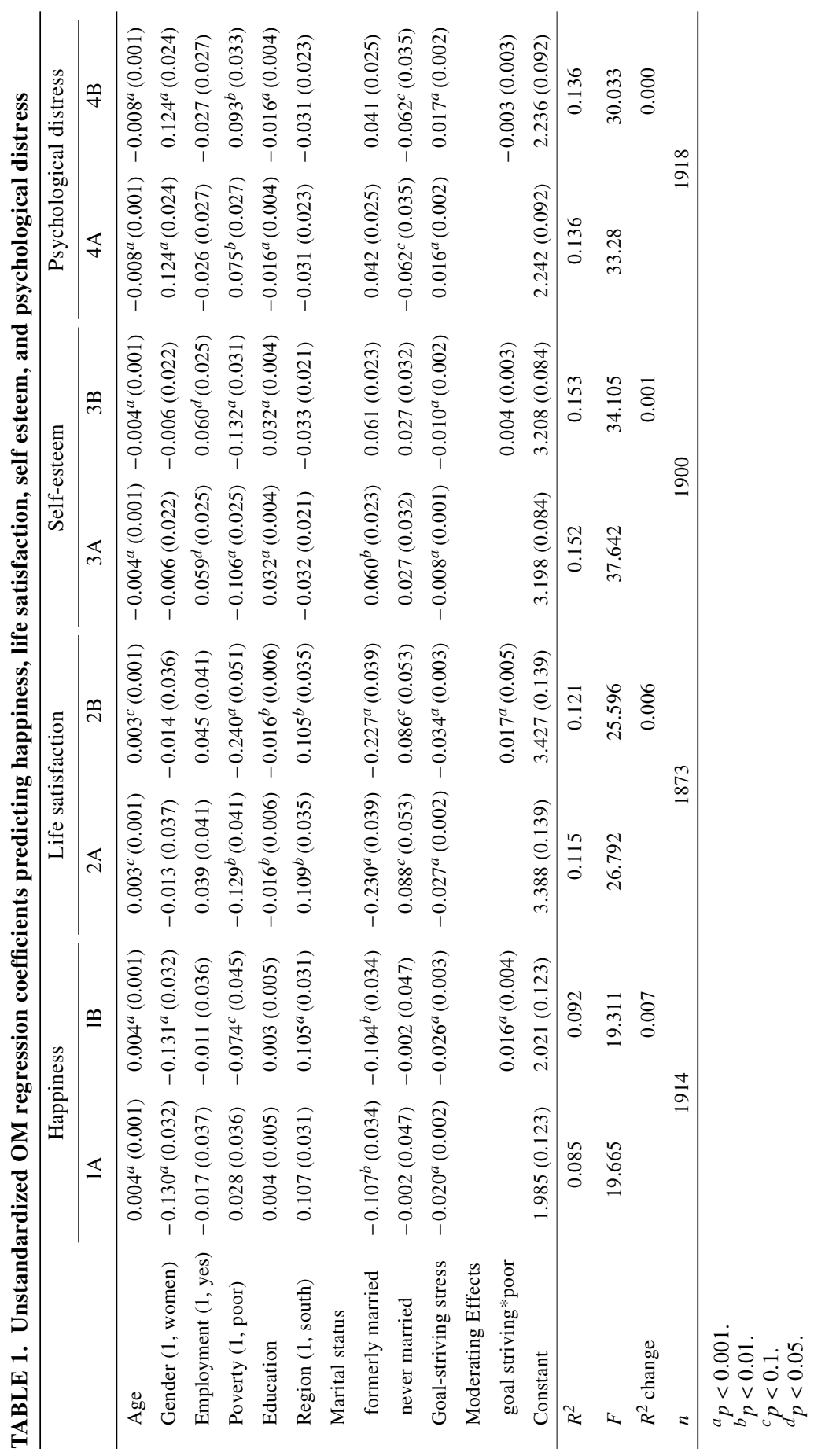



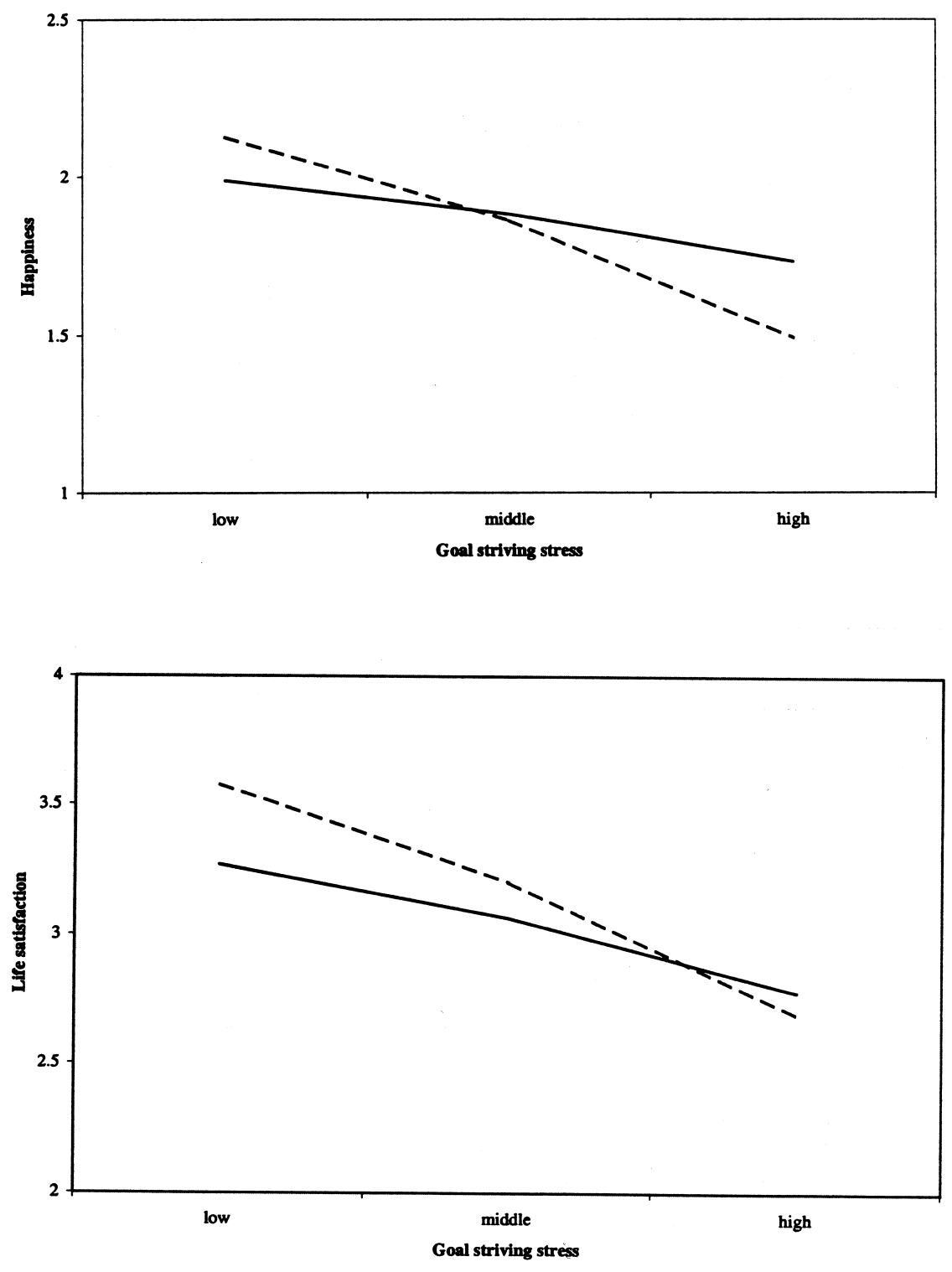

FIGURE 1. Interaction between poverty status and goal-striving stress: — - poor; $-{ }_{-}--$, not poor. 
"where step 10 represents your best way of life and step 1 represents your worst way of life." Aspirations were measured by asking the step number that best described where the respondent would like to be the following year. Achievement was measured by asking the step number that best described where the respondent was at the current time. Importance of the goal was measured by asking respondents, on a fourpoint scale, how disappointed they would be if they could never reach the step to which they aspire.

Analytic strategy

Ordinary least squares regression was used to estimate the effects of goal striving stress on a set of mental health outcomes and to examine the moderating effects of poverty status on the relationships between goal-striving stress and mental health. In all analyses, the effects of age (years), education (in years completed), gender, and marital and employment status were controlled. Analyses were weighted up to the original 2,107 cases.

\section{RESULTS}

As shown in columns "A" of TABLE 1, goal-striving stress is strongly related to mental health: As goal-striving stress increases, levels of happiness, life satisfaction, and self-esteem decrease; and levels of psychological distress increase. The moderating effects of poverty status are presented in columns "B." Although no moderating effects were found for self-esteem and psychological distress, effects were found for well-being. Compared to poor persons, individuals above poverty had higher levels of happiness and life satisfaction at low levels of goal striving, but as levels of striving increased, individuals above poverty had significantly lower levels of happiness and life satisfaction. FIGURE 1 illustrates these findings.

\section{DISCUSSION}

Finding that goal-striving stress is negatively related to mental health among black Americans is consistent with arguments that locate the genesis of illness within the social structure. Goal striving appears to be a source of chronic stress; for blacks, perhaps it is particularly pernicious because racial prejudice and discrimination systematically block opportunities to achieve life goals. The moderating effects of poverty status support this speculation. Perhaps those above poverty attach their wellbeing to economic advancement and, having achieved a measure of success, assume that additional effort will yield additional reward. In a society in which rewards are often distributed inequitably, this assumption can lead to decreased well-being. Poverty status did not moderate the relationship between goal-striving stress and selfesteem or psychological distress. However, if the mismatch between efforts and rewards persists, more severe mental health problems, such as depression and suicide, may develop. It is likely that the impact of goal-striving stress varies over time and across the life course. In addition to consideration of temporal relationship between goal-striving stress and health, future research should consider racially differ- 
entiated health outcomes such as hypertension and the interplay between goalstriving stress and coping strategies such as John Henryism.

\section{REFERENCES}

1. Aneshensel, C. 1992. Social stress: theory and research. Annu. Rev. Sociol. 18: 1538.

2. DRESSLER, W. 1988. Social consistency and psychological distress. JHSB 29: 79-91.

3. JACKSON, J. et al. 1996. Perceptions and experiences of racism and the physical and mental health status of African Americans: a thirteen-year national panel study. Ethnic Dis. 6: 123-138.

4. Neighbors, H. \& S. Lumpkin. 1990. The epidemiology of mental disorder in the black population. In Handbook of Mental Health and Mental Disorder among Black Americans. Dorothy Ruiz, Ed. Greenwood Press, New York.

5. Parker, S. \& R. Kleiner. 1966. Mental illness in the urban Negro community. Free Press, New York. 